\title{
Antistreptolysin O Titers: Normal Values for Children Ages 5 to 15 at Debre Berhan Referral Hospital, Ethiopia
}

\author{
Tsegahun Asfaw, Demissew Shenkute, Mihret Tilahun, Nigus Zegeye
}

Department of Medical Laboratory Science, Debre Berhan University, Debre Berhan, Ethiopia

\section{Email address:}

tsegahun.asfaw12@gmail.com(T. Asfaw),demissewshen@gmail.com(D. Shenkute), tilahunmihret21@gmail.com(M. Tilahun), kingster2002@gmail.com(N.Zegeye)

\section{To cite this article:}

Tsegahun Asfaw, Demissew Shenkute, Mihret Tilahun, Nigus Zegeye. Antistreptolysin O Titers: Normal Values for Children Ages 5 to 15 at Debre Berhan Referral Hospital, Ethiopia. Journal of Family Medicine and Health Care. Vol. 4, No. 1, 2018, pp. 1-4.

doi: $10.11648 /$ j.jfmhc.20180401.11

Received: February 15, 2018; Accepted: March 27, 2018; Published: April 20, 2018

\begin{abstract}
Anti-streptolysin O (ASO) titer assists for the diagnosis of Group A, Group C and Group G streptococcal infections and their sequele. Reference value of Anti-streptolysin O titer is not available for Ethiopian populations. This study determined the upper limit of normal reference value in apparently healthy children. Participants with a history of streptococal disease were excluded. A total of 127 blood samples were collected from 127 participants with age range of 5-15 years. Serum was used to determine Anti-Streptolysin O-titers. The average ASO Upper limits of Normal (ULN) titer for the total participants was $360 \mathrm{IU} / \mathrm{ml}$ with a median $200 \mathrm{IU} / \mathrm{ml}$. The ASO ULN for both male and female children was $320 \mathrm{IU} / \mathrm{ml}$ with a median of $200 \mathrm{IU} / \mathrm{ml}$. The highest ASO ULN was observed for the age group of 9-12 years (400 IU/ml with median of 200 $\mathrm{IU} / \mathrm{ml}$ ) followed by $360 \mathrm{IU} / \mathrm{ml}$ for the age group 5-8 years and age group 13-15 years with a median of $200 \mathrm{IU} / \mathrm{ml}$. This finding shows that ASO ULN are similar to those reported in countries with different climates and populations. Package inserts interpreting ASO titer $>400 \mathrm{IU} / \mathrm{ml}$ as recent streptococcal infection is applicable for Ethiopian population.
\end{abstract}

Keywords: Children, Apparently Healthy, ASO Titers, Ethiopia

\section{Introduction}

Rheumatic heart disease (RHD) and Rheumatic fever (RF) remain significant causes of cardiovascular disease and mortality in children particularly in the developing countries [1]. RF is estimated to affect 20 million people and is a leading cause of death during the first five decades of life [2]. It is endemic in developing countries with annual incidence of 100-200 cases per 100,000 school aged children [3]. The fact that RF and RHD are theoretically preventable but still remains a problem in developing countries. Reason most advanced is that these countries cannot afford the economic and social cost involved [2]. RHD and RF are an autoimmune disease that follows infection with GAS; however, the isolation of GAS is uncommon $(<15 \%)$, and so confirmation of the diagnosis often relies on streptococcal antibody tests [4]; the most frequently performed tests are anti-streptolysin O (ASO) titer [5]. If possible, it is recommended that the titer be determined in the acute phase and then determined in the convalescent phase 14 to 28 days later, with a positive result defined as a rise in titer of twofold or more [4]. However, it is not always practicable to obtain a second sample for titer determination, particularly in developing countries, where acute rheumatic heart Disease is the most common. Therefore, it is generally accepted that if only a single specimen is available, a titer greater than the upper limit of normal at the initial testing can be considered presumptive evidence of a preceding streptococcal infection [6-8]. However, there has been no investigation of upper limit of normal reference values for populations in Ethiopia despite of, RHD is the number one cardiac problem in children with a prevalence rate of 4.6-7.1per 1000 [9-10]. Therefore this study is designed to establish upper limit of normal reference value of ASO titers in apparently healthy children with the age range of 5-15 years.

\section{Materials and Methods}

\subsection{Study Design and Setting}

Cross sectional study was conducted among 127 apparently healthy children (5-15 years) who are devoid of 
streptococcal infections attending pediatric clinic of DebreBerhan referral hospital from 2016- September, 2017. DebreBerhan referral hospital is located in the Semen Shewa Zone of the Amhara Region, $130 \mathrm{Kms}$ Northeast of Addis Ababa.

\subsection{Sample Collection, Handling, Transport and Analysis}

Blood was collected from each study participant by trained nurse. A total of $3 \mathrm{ml}$ of venous blood was collected using sterile $5 \mathrm{ml}$ syringe and immediately transferred to a glass tube without anticoagulant. It was transported to DebreBerhan university medical microbiology laboratory and then serum was separated at 3000 RPM for 10 minutes for ASO titer determination. The demographic data was collected using a questionnaire. All expected factors were included in the questionnaire and clinical information was obtained from all patients by reviewing medical records. Patients with incomplete data were excluded.

\subsection{Determination of Anti-Streptolysin-O (ASO) Titer}

Anti-Streptolysin O- latex reagents (LiNEAR Chemicals. s. L, Spain) were used to determine ASO titer. All test reagents and serum samples were brought to room temperature before the test was done. Normal saline $(0.85 \%$ $\mathrm{NaCl}$ ) was used for dilution purpose. Fifty micro liter of serum and one drop of the ASLO-Latex antigen were mixed using disposable stirrer on a circle on the card and rotated on the mechanical rotator (100 RPM) for 2 minutes. Both positive and negative controls were run in parallel. It was observed under visible light source for any degree of agglutination. When the test becomes reactive, $50 \mu \mathrm{L}$ of the serum diluted with $50,100,150,200 \mu \mathrm{L}$ of saline etc to make it simple dilution (undiluted, 1/2,1/3,1/4, 1/5 etc) until the result became non-reactive. The serum ASO concentration could then be calculated approximately by multiplying the dilution factor (i.e. 2, 3, 4 or 5) by the detection limit. This method was chosen because it is the widely used technique for ASOT measurement in Ethiopia.

\subsection{Data Quality Control Measures}

Standard Operational Procedures were strictly followed during collection, preservation and transportation of blood specimens.

\subsection{Data Analysis}

Mean, standard deviation of the mean and the median for ASO serum levels among the tested participants were calculated to determine the significance of the differences of these values among different sex and age groups. As the standard definition of a reference range for ASO measurement is defined by separating the upper $20 \%$ from the lower $80 \%$ of the group distribution in a dichotomous fashion $[7,8,11]$. The choice of the 80th centile cutoff rather than more traditional upper-limit-of-normal calculations is based upon studies that found that more than 80 to $90 \%$ of patients with acute rheumatic fever have streptococcal titers that are above the 80th centile for the healthy controls with no clinical evidence of recent streptococcal infection $[8,11]$. So, it is assumed that in any population a proportion of apparently healthy individuals will have had a recent, subclinical GAS infection [11].

\subsection{Ethical Considerations}

Ethical approval was obtained by DebreBrehan university research and review committee and official permission was obtained from head department of DebreBrehan referral hospital. Written informed consent was obtained from each child's parent or guardian and assent from study participants older than 12 years.

\section{Results}

\subsection{Socio-Demographic Characteristics of Study Participants}

Among 127 participants, 68 (53.5\%) were males and 59 $(46.5 \%)$ were females. The children were symmetrically distributed in age groups, $42(33.1 \%)$ were in age group of 58 years, $43(33.8 \%)$ were in age group of $9-12$ years and 42 $(33.1 \%)$ were in age group of $13-15$ years

Table 1. The age and sex distribution of children tested for ASO normal value levels.

\begin{tabular}{ll}
\hline Characteristics & Frequency (No. (\%) of participants) \\
\hline Gender & $68(53.5)$ \\
Male & $59(46.5)$ \\
Female & \\
Age (Year) & $42(33.1)$ \\
$5-8$ & $43(33.8)$ \\
$9-12$ & $42(33.1)$ \\
$13-15$ & \\
\hline
\end{tabular}

\subsection{Antistreptolysin O (ASO) Titer Analysis}

Table 2 shows the median and cut of 80 percentile upperlimit of normal reference values for ASO titer according to sex and age groups of enrolled subjects. The ASO ULN for the total subjects was $360 \mathrm{IU} / \mathrm{ml}$ with a median $200 \mathrm{IU} / \mathrm{ml}$. The ASO ULN for both male and female children were 320 $\mathrm{IU} / \mathrm{ml}$ with a median of $200 \mathrm{IU} / \mathrm{ml}$. The highest ASO ULN was observed for the age group of 9-12 years $(400 \mathrm{IU} / \mathrm{ml}$ with median of $200 \mathrm{IU} / \mathrm{ml}$ ) followed by $360 \mathrm{IU} / \mathrm{ml}$ for the age group 5-8 years and age group 13-15 years with a median of $200 \mathrm{IU} / \mathrm{ml}$. 
Table 2. The cut of 80 percentile upper limit of normal reference values for ASO titer by sex and age groups for children 5-15 years.

\begin{tabular}{lllll}
\hline \multirow{2}{*}{ Characteristics } & No. (\%) of participants & ASO (IU/ml) & Median & $\mathbf{8 0 \%}$ upper limit of normal \\
\cline { 3 - 5 } & & Mean \pm SD & & 320 \\
\hline Sex & $68(53.5)$ & $245.2 \pm 99.5$ & 200 & 320 \\
Male & $49(46.5)$ & $244.4 \pm 96.9$ & 200 & 360 \\
Female & & & & 400 \\
Age & $42(33.1)$ & $240 \pm 81.02$ & 200 & 360 \\
$5-8$ & $43(33.8)$ & $266.6 \pm 96.6$ & 200 & 360 \\
$9-12$ & $42(25.2)$ & $240 \pm 82.8$ & 200 & 200 \\
$13-15$ & $127(100)$ & $243.6 \pm 91.8$ & & \\
Total & & &
\end{tabular}

\section{Discussion}

The present study was designed to establish normal range of ASO titers in apparently healthy individuals among different age groups. In present study a total of 127 children with the age of 5-15 years, $68(53.5 \%)$ male and $59(46.5 \%)$ were conveniently selected and investigated for the ASO ULN. The ASO ULN for the total subjects was $360 \mathrm{IU} / \mathrm{ml}$ with a median $200 \mathrm{IU} / \mathrm{ml}$. The ASO ULN for both male and female children were $320 \mathrm{IU} / \mathrm{ml}$ with a median of $200 \mathrm{IU} / \mathrm{ml}$. The highest ASO ULN was observed for the age group of 912 years $(400 \mathrm{IU} / \mathrm{ml}$ with median of $200 \mathrm{IU} / \mathrm{ml})$ followed by $360 \mathrm{IU} / \mathrm{ml}$ for the age group 5-8 years and age group 13-15 years with a median of $200 \mathrm{IU} / \mathrm{ml}$. The ASO ULN in this study were found to be roughly similar to those reported from other regions. In comparison with data from the Minnesota, USA (333 IU/ml) [8], Mumbai, India (305 IU/ml) [12], Australia (320 IU/ml) [13] and Korea (326 IU/ml) [14]. Relatively higher ASO ULN was obtained from study conducted in Sana'a, Yemen (276.2 IU/ml) [15], Fiji (276 $\mathrm{IU} / \mathrm{ml})$ [16], USA (240 IU/ml) [17], Tanzania (200 IU/ml) [18], Sweden (200 IU/ml) [19] and in a different regions of India $(239 \mathrm{IU} / \mathrm{ml})[20]$. However, lower result was obtained from study conducted in Egypt (400IU/ml) [21].

Most of these values exceeded the normal level set by laboratories which is $200 \mathrm{IU} / \mathrm{mL}$. The higher ASO titers in Yemen and Australia, Fiji and India are probably due to the fact that tonsillitis and impetigo are endemic particularly in children [16, 22]. In Ethiopia, RHD is the number one cardiac problem in children with a prevalence rate of 4.6-7.1 per $1000[9,5]$ suggest that there is high prevalence of tonsillitis and pharyngitis. Although children with a recent history of tonsillitis and impetigo were excluded, the ASO titers remain elevated for many months; hence, some children, whose ASO titers might be in the process of returning to their baseline level after a case of tonsillitis or impetigo were included [19].

By applying the simple non parametric method that has been used in previous studies to analyze the present study data, the cutoff values were found similar to those obtained by the parametric method. However, the parametric method for data analysis that was used in this study has some advantages over the non-parametric method. The nonparametric method often produces unlikely irregular patterns in the centiles with age, unless a large sample is used and wide age intervals are specified [16]. The results may be artificially affected by the choice of age groups, especially when titers have a complex pattern of change with age. This study will recommend that Ethiopians clinicians use the cutoff values for single upper limit of normal cutoff value for children aged from 5 to 15 years, rather than sub-age groups, such as $5-8$ years, 9 to 12 years and 13 to 15 . This is because there is minor variability in the year by year values that was found in children aged 5 to 15 years. No significant association presents between sex and ASO concentrations, suggesting that the female cut off equal to the male cut off. This issue has not been discussed by other researchers previously.

\section{Conclusions}

This finding showed that ASO ULN are similar to those reported in countries with different climates and populations. Package inserts interpreting ASO titer $>400$ $\mathrm{IU} / \mathrm{ml}$ as recent streptococcal infection is applicable for Ethiopian population.

\section{Conflict of Interest}

The authors declare that they have no conflict interests.

\section{Funding}

This work was funded from DebreBerhan University core budget.

\section{Author Contributions}

TA and NZ participated in its design. TA, MT, and DS performed the laboratory activities. TA analyzed the data and wrote the manuscript. NZ reviewed the manuscript. All authors read and approved the final manuscript.

\section{Acknowledgements}

We would like to thank nurse staffs and head director of DebreBerhan referral hospital for their help during data collection process. 


\section{References}

[1] Parks T, Smeesters PR, Curtis N, Steer AC. ASO titer or not? When to use streptococcal serology: a guide for clinicians. Eur J ClinMicrobiol Infect Dis. 2015; 34: 845-849.

[2] Mandor BI, Asuquo AE, Abia-Bassey L, Benjamin TP, Akpan IA Etuk IS, Eyong ME, Meremiku MM. Antistreptolysin O (ASO) titers and beta-haemolytic streptococcus (BHS) in Children in Calabar, Nigeria. IOSR Journal of Nursing and Health Science. (Jul - Aug 2013); 1 (5): 42-46.

[3] Batzloff M, Yan H, Davies M, et al. Preclinical Evaluation of a Vaccine Based on Conseved Region of $\mathrm{M}$ protein that prevents group A Streptococcal Infection, Indian Journal of medical Research, 119suppl, 2004, 104-107.

[4] Andrew CS, Suzanna V, Roselyn R, Joseph K. Michael B. Adam WJ, et al., Normal Ranges of Streptococcal Antibody Titers Are Similar Whether Streptococci Are Endemic to the Setting or Not. Clinical and Vaccine Immunology, Feb. 2009; 16 (5): 172-175.

[5] Shet A and Kaplan EL. Clinical use and interpretation of group A streptococcal antibody tests: a practical approach for the pediatrician or primary care physician. Pediatr. Infect. Dis. J. 2002; 21:420-426.

[6] Kaplan EL, Top FH, Dudding BA, and Wannamaker LW. Diagnosis of streptococcal pharyngitis: differentiation of active infection from the carrier state in the symptomatic child. J. Infect. Dis. 1971; 123:490-501.

[7] Klein GC, Baker CN, and Jones WL. "Upper limits of normal" antistreptolysin $\mathrm{O}$ and antideoxyribonuclease B titers. Appl. Microbiol. 1971, 21: 999-1001.

[8] Wannamaker LW and Ayoub EM. Antibody titers in acute rheumatic fever. Circulation 1960; 21:598-614.

[9] Oli K, Porteous J. Prevalence of rheumatic heart disease among school children in Addis Ababa. East Afr Med J. 1999; 76:601-5.

[10] Oli K, Tekle-Haimanot R, Forsgren L, Ekstedt J. Rheumatic heart disease prevalence among schoolchildren of an Ethiopian rural town. Cardiology. 1992; 80 (2):152-5.

[11] Ayoub EM and Wannamaker LW. Evaluation of the streptococcal deoxyribonuclease B and diphosphopyridine nucleotide antibody tests in acute rheumatic fever and acute glomerulonephritis. Pediatrics. 1962; 29:527-538.
[12] Karmarkar MG, Venugopal V, Joshi L, and Kamboj R. Evaluation \& revaluation of upper limits of normal values of anti-streptolysin $\mathrm{O}$ and ant-deoxyribonuclease B in Mumbai. Indian J. Med. Res. 2004; 119 (Suppl.):26-28.

[13] Danchin, MH, Carlin JB, Devenish W, Nolan TM, and Carapetis JR. 2004. New normal ranges of antistreptolysin O and anti-deoxyribonuclease B titres for Australian children. J. Paediatr. Child Health41:583-586.

[14] Kim S. and Lee YN. Asymptomatic infection by Streptococcus pyogenes in school children and diagnostic usefulness of antideoxyribonuclease B. J Korean Med Sci. $2005 ; 20: 938-40$.

[15] Khaled AA and Hassan AA. Anti Streptolysin O; Normal Values for Healthy Children Aged from 5 to 15 Years Old in Sana'a City-Yemen. Annals of Clinical and Laboratory Research. 2015; Vol. 3 No. 1:1.

[16] Steer AC, Carapetis JR, Nolan TM, Shann F. Systematic review of rheumatic heart disease prevalence in children in developing countries: the role of environmental factors. J Paediatr Child Health. 2002; 38: 229-234.

[17] Kaplan EL, Rothermel CD, and Johnson DR. Antistreptolysin $\mathrm{O}$ and anti-deoxyribonuclease B titers: normal values for children ages 2 to 12 in the United States. Pediatrics. 1998; 101:86-88.

[18] Mhalu FS and Matre R. Antistreptolysin O and antideoxyribonuclease $\mathrm{B}$ titres in blood donors and in patients with features of nonsuppurative sequelae of group A streptococcus infection in Tanzania. E Afr Med J. 1995; 72:33-6.

[19] Nimmo GR, Tinniswood RD, Nuttall N, Baker GM, and McDonald B. Group A streptococcal infection in an aboriginal community. Med. J. Aust. 1992; 157:521-522.

[20] Sethi S, Kaushik K, Mohandas K, Sengupta C, Singh S, and Sharma M. Anti-streptinolysin O titres in normal healthy children aged 5-15. Indian Pediatr. 2003: 40:1068-1071.

[21] Alyaa Amal Kotby, Nevin Mamdouh Habeeb and Sahar Ezz El Elarab Antistreptolysin O titer in health and disease: levels and significance. Pediatric Reports 2012; 4:e8.

[22] Yemen MHP. Newsletter- supply 13. Medical Statistical Department, Ministry of Health and Population, RO Y. 2013. 